\title{
Shack-Hartmann sensor based optical quality testing of whole slide imaging systems for digital pathology
}

\author{
S. Mojtaba Shakeri*a ${ }^{*}$ Bas Hulsken ${ }^{\mathrm{b}}$, Lucas J. van Vliet ${ }^{\mathrm{a}}$, Sjoerd Stallinga ${ }^{\mathrm{a}}$ \\ ${ }^{a}$ Quantitative Imaging Group, Department of Imaging Physics, Delft University of Technology, \\ Lorentzweg 1, 2628 CJ, Delft, The Netherlands; ${ }^{b}$ Philips Digital Pathology, Veenpluis 6, 5684 PC \\ Best, The Netherlands
}

\begin{abstract}
Whole Slide Imaging (WSI) systems are used in the emerging field of digital pathology for capturing high-resolution images of tissue slides at high throughput. We present a technique to measure the optical aberrations of WSI systems using a Shack-Hartmann wavefront sensor as a function of field position. The resulting full-field aberration maps for the lowest order astigmatism and coma are analyzed using nodal aberration theory. According to this theory two coefficients describe the astigmatism and coma inherent to the optical design and another six coefficients are needed to describe the cumulative effects of all possible misalignments on astigmatism and coma. The nodal aberration theory appears to fit well to the experimental data. We have measured and analyzed the full-field aberration maps for two different objective lens-tube lens assemblies and found that only the optical design related astigmatism coefficient differed substantially between the two cases, but in agreement with expectations. We have also studied full-field aberration maps for intentional decenter and tilt and found that these affect the misalignment coefficient for constant coma (decenter) and the misalignment coefficient for linear astigmatism (tilt), while keeping all other nodal aberration theory coefficients constant.
\end{abstract}

Keywords: Digital pathology, Whole Slide Imaging, Shack-Hartmann wavefront sensor, Optical Transfer Function, nodal aberration theory, astigmatism, coma

\section{INTRODUCTION}

Digital pathology is based on the use of digital images of tissues for diagnosis of disease. These images are typically $\sim 15$ $\mathrm{mm}^{2}$ in size and are sampled at a sampling density of $\sim 0.25 \mu \mathrm{m} /$ pixel with a Whole Slide Imaging (WSI) system. This "pushbroom" scanner sweeps the slide lane by lane ( $\sim 1 \mathrm{~mm}$ wide) and stitches the lanes into the final high-resolution image (Figure 1a). It is important in assembly and maintenance to test and monitor the optical quality of WSI systems. This can be done by analyzing through-focus images of custom test targets for measuring the Optical Transfer Function (OTF) and subsequently extracting the different primary aberration coefficients [1]. This noninvasive testing method provides a systematic approach to quantify the optical quality solely by using a custom-built resolution target and a software tool. However, this OTF-based method needs validation using direct aberration measurements. A well-known method is to extract aberrations from wavefront measurements using a Shack-Hartmann sensor [2]. The aberrations that are so revealed are due to imperfections in the individual components and also due to possible alignment errors in the system. The wavefront measurements with the Shack-Hartmann sensor can be done for different positions in the Field Of View (FOV) of the objective lens. The measurement of this full-field aberration map can be used to reveal the root cause of the aberrations. This could subsequently be used to modify the alignment of the optical system in such a way as to minimize the overall level of aberrations or for testing the quality of individual optical components (objective lens, tube lens). Several studies have been carried out to develop a theory of misalignment effects on the full-field aberration maps, mostly for aligning telescopes for astronomy applications [3-6]. Here, we present details of a technique to adapt the Shack-Hartmann test for measuring the full-field aberration map of a WSI system, and we present an analysis of the fullfield aberration maps for revealing different misalignment root causes such as decenter and tilt of optical components.

The outline of this paper is as follows. In section 2, we describe the Shack-Hartmann measurement setup and the theory of full-field aberration map analysis for coma and astigmatism. Then we present the comparison of these full-field aberration maps to the OTF-based aberration test for two different objective lens-tube lens assemblies in section 3 . We

Design and Quality for Biomedical Technologies VIII, edited by Ramesh Raghavachari, Rongguang Liang Proc. of SPIE Vol. 9315, 931504 - (C) 2015 SPIE · CCC code: 1605-7422/15/\$18 · doi: 10.1117/12.2077085 
also apply the full-field aberration map analysis to a configuration with intentional decenter and tilt misalignment. Finally, the paper is concluded with a brief summary in section 4 .

\section{METHODS}

\subsection{Shack-Hartmann test setup}

We built a wavefront sensing setup using an Optocraft SHR-150-CL Shack-Hartmann sensor (12 bit, detection area $11.8 \times 8.9 \mathrm{~mm}^{2}, 78 \times 59$ microlens array) to measure the aberrations of the optical system under inspection (Figure $1 \mathrm{~b}$ ). A pinhole $(5 \mu \mathrm{m}$ diameter) mounted in between a glass slide and a coverslip is used as a point source object. In order to provide a (near) parallel wavefront to the SH sensor, an additional collimating lens (Melles Griot 01LAO536 - 120mm focal length) is placed at its focal distance from the system's image plane. We measured the aberrations of this collimating lens separately to account for this effect in the final results. The aberrations for different positions in the FOV of the objective lens can be probed by laterally translating the pinhole slide.

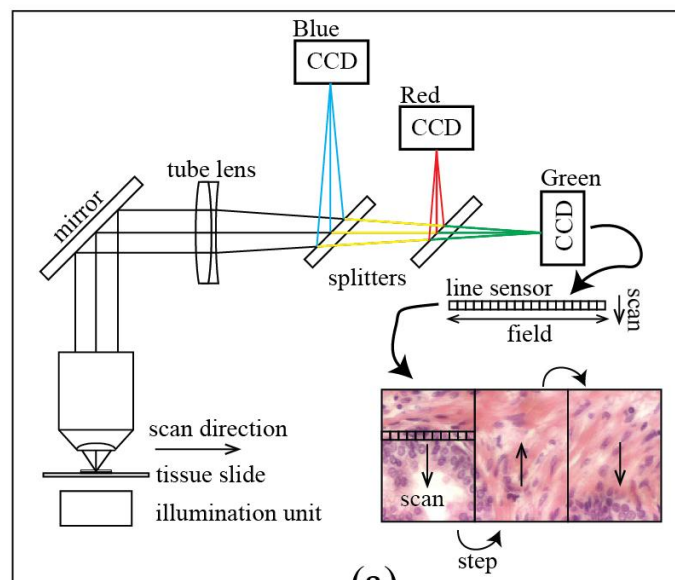

(a)

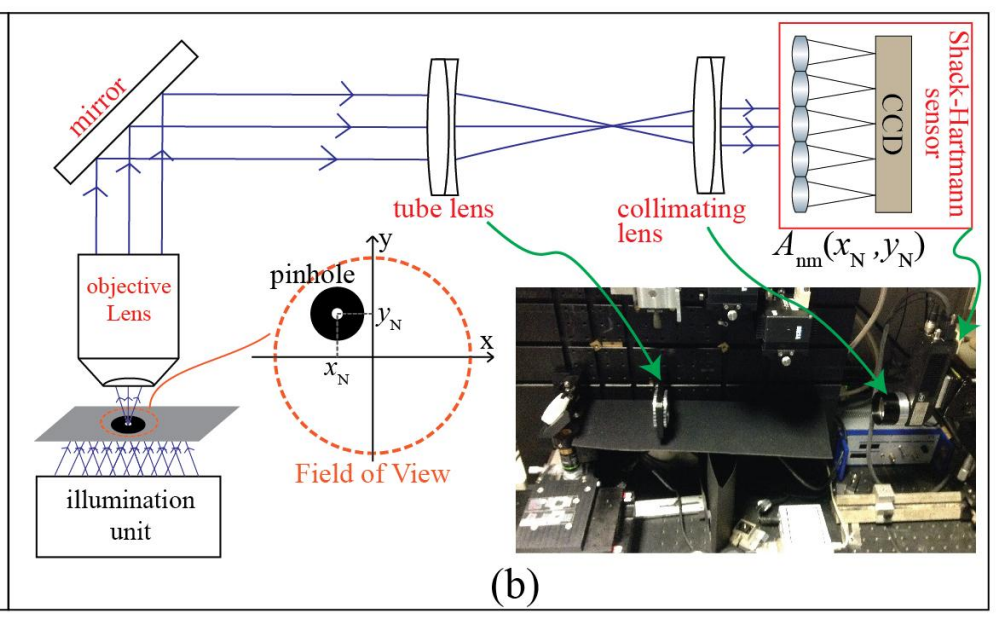

(b)

Figure 1. Schematic layout of the (a) home-built line sensor based WSI system, (b) Shack-Hartmann test setup. The pinhole can be moved in the field of view and then the corresponding aberration coefficients $A_{\mathrm{nm}}\left(x_{\mathrm{N}}, y_{\mathrm{N}}\right)$ are measured by the ShackHartmann sensor.

The alignment procedure is depicted step by step in Figure 2. The alignment of the objective lens, folding mirror and tube lens are based on the alignment of two mutually perpendicular reference laser lines. These reference laser lines are aligned with respect to a mechanical reference, namely the mounting plate with an array of grooves for mounting the different optical components. Any residual deviations from perpendicularity arise from the mechanical adjustment error of the irises used to align the reference laser lines. The measurement of the aberrations is started by placing the pinhole at the center of the FOV (following step VI in Figure 2). The Shack-Hartmann sensor is translated to be perpendicular to the incident wavefront after moving the pinhole to each new position in the FOV, so that the measured coefficients for tilt and defocus become as small as possible. The measurements of the aberrations were restricted to positions up to about $80 \%$ of the full FOV diameter in view of mechanical constraints of the objective lens mount and Shack-Hartmann sensor mount.

\subsection{Full-field aberration map analysis}

Thompson in [7-9] developed a new way of analyzing aberrations in an optical system by looking into the field dependency of each individual aberration. He showed that for well-aligned rotationally symmetrical optical systems, the full-field aberration map is rotationally symmetric as well. Any misalignment breaks the symmetry of the map. For each aberration type the symmetry is broken in a specific way, giving rise to a limited set of nodes (points in the FOV with zero aberration of the considered type) in the full-field aberration map. The map will have one node at the center of the FOV if the optical system is well aligned. The maximum number of nodes for each type of aberration is equal to the power of the field dependency for that aberration, i.e. if the aberration scales as $\sim \eta^{p}$, with $\eta$ a field coordinate, then there will be at most $p$ nodes in the full-field aberration map. For example, for lowest order astigmatism we have $p=2$, implying that any misalignment can create at maximum two nodes in the full-field aberration map. This nodal aberration 
theory has been used by Tessieres and Manuel in unpublished manuscripts [3, 10] to relate the full-field Zernike coefficient map to the presence of possible misalignment in reflective optical systems such as telescopes. We use this theory to study misalignment effects on (lowest order order) coma and astigmatism, as these are critical to the optical quality of WSI systems [1].

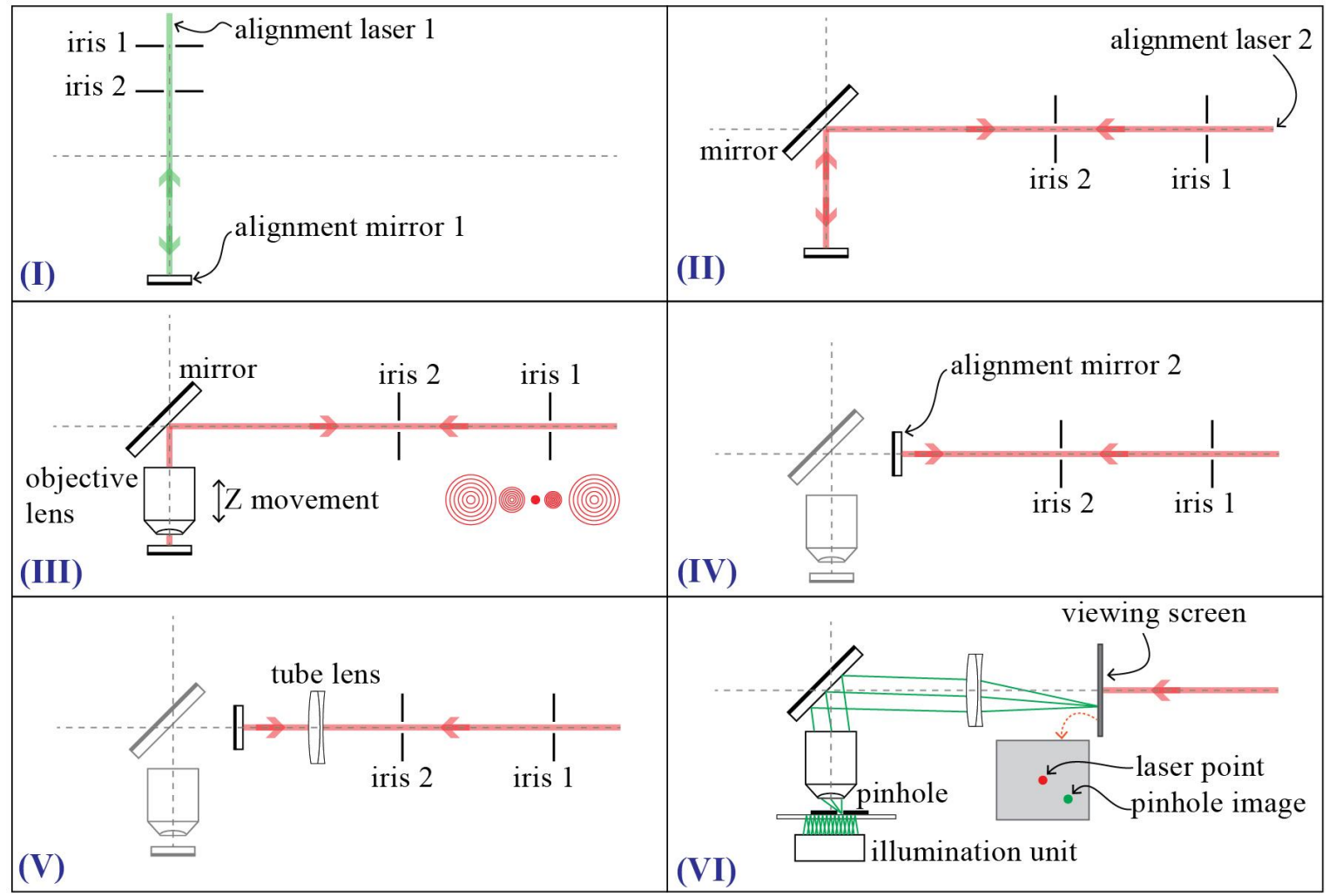

Figure 2. Alignment procedure. Step (I) is to align mirror 1 perpendicular to laser 1 reference line. The mirror is placed such that both laser light and its reflection pass through the irises. Step (II) is to place the folding mirror at 45 deg with respect to laser 2 reference line. Step (III) is to align the objective lens. To do so, the objective lens will be moved along the z-axis. If the optical axis of the objective lens is aligned with the reference laser line then the through-focus spots are concentric. The size of the irises needs to be readjusted in order to see the fringes at different distances. Step (IV) is to place another alignment mirror perpendicular to the laser 2 reference line in order to align the tube lens. In step (V), the tube lens is positioned such that the reflected laser light passes through the two irises and there is no double reflection pattern on the left side of the iris. Now, the three optical components are aligned. Step (VI) is to place the pinhole at the optical axis. At this step the alignment mirrors are removed and the illumination unit is placed bellow the objective lens. A semi-transparent viewing screen (e.g. thin paper) is used to visually monitor the position of the pinhole image and laser reference line. The pinhole slide is translated until its image coincides with the laser point at the viewing screen.

The aberration function of the optical system can be expanded in Zernike polynomials of radial order $n$ and azimuthal order $m$, where the coefficients are functions of the field coordinates $x$ and $y[3,10]$ :

$$
W=\sum_{n, m} A_{n m}(\mathrm{x}, \mathrm{y}) Z_{n m}(\rho, \varphi)
$$

where $\rho$ and $\varphi$ are the radial and azimuthal coordinates of the (circular and normalized) exit pupil. From now on we will focus on (lowest order) coma and astigmatism only. In an aligned centrosymmetric optical system these have, to the lowest order, a linear and quadratic dependency on the field coordinates, respectively:

$$
\begin{aligned}
W_{\text {astigmatism }} & =A_{22}(\mathrm{x}, \mathrm{y}) Z_{22}+A_{2-2}(\mathrm{x}, \mathrm{y}) Z_{2-2}=\left(a_{\text {quadratic }}\left(\mathrm{x}^{2}-\mathrm{y}^{2}\right)\right) Z_{22}+\left(2 a_{\text {quadratic }} x y\right) Z_{2-2}, \\
W_{\text {coma }} & =A_{31}(\mathrm{x}, \mathrm{y}) Z_{31}+A_{3-1}(\mathrm{x}, \mathrm{y}) Z_{3-1}=\left(c_{\text {linear }} x\right) Z_{31}+\left(c_{\text {linear }} y\right) Z_{3-1},
\end{aligned}
$$

where $a_{\text {quadratic }}$ and $c_{\text {linear }}$ are constants inherent to the optical design of the system for astigmatism and coma respectively. For example, $c_{\text {linear }}$ is a measure for the Offense against the Sine Condition (OSC). If the optical system is misaligned, 
i.e. if the rotational symmetry around the optical axis is broken, then the field dependency of the Zernike aberration coefficients $A_{n m}$ changes as follows [10]:

$$
\begin{aligned}
A_{22}(x, y) & =a_{\text {quadratic }}\left(x^{2}-y^{2}\right)-a_{y L \text { inear }} y+a_{x L \text { inear }} x+a_{h \text { Constant }}, \\
A_{2-2}(x, y) & =2 a_{\text {quadratic }} x y+a_{y L \text { inear }} x+a_{x L \text { inear }} y+a_{d \text { Constant }}, \\
A_{31}(x, y) & =c_{\text {linear }} x+c_{x \text { Constant }}, \\
A_{3-1}(x, y) & =c_{\text {linear }} y+c_{y \text { Constant }},
\end{aligned}
$$

where the misalignments introduce constant coma, constant astigmatism and linear astigmatism. The coefficients of all contributions to the full-field aberration map can be found using a least-squares fit. This can be done by solving the following set of equations for $a$ and $c$ by measuring $A_{n m}$ at $N$ points in field of view:

$$
\begin{aligned}
& \left(\begin{array}{c}
A_{31}^{1} \\
A_{3-1}^{1} \\
\vdots \\
A_{31}^{N} \\
A_{3-1}^{N}
\end{array}\right)=\left(\begin{array}{ccc}
x_{1} & 0 & 1 \\
y_{1} & 1 & 0 \\
\vdots & \vdots & \vdots \\
x_{N} & 0 & 1 \\
y_{N} & 1 & 0
\end{array}\right)\left(\begin{array}{c}
c_{\text {linear }} \\
c_{y \text { Constant }} \\
c_{x \text { Constant }}
\end{array}\right) \\
& \left(\begin{array}{c}
A_{22}^{1} \\
A_{2-2}^{1} \\
\vdots \\
A_{22}^{N} \\
A_{2-2}^{N}
\end{array}\right)=\left(\begin{array}{ccccc}
\left(x_{1}\right)^{2}-\left(y_{1}\right)^{2} & -y_{1} & x_{1} & 0 & 1 \\
2 x_{1} y_{1} & x_{1} & y_{1} & 1 & 0 \\
\vdots & \vdots & \vdots & \vdots & \vdots \\
\left(x_{N}\right)^{2}-\left(y_{N}\right)^{2} & -y_{N} & x_{N} & 0 & 1 \\
2 x_{N} y_{N} & x_{N} & y_{N} & 1 & 0
\end{array}\right)\left(\begin{array}{c}
a_{\text {quadratic }} \\
a_{\text {yLinear }} \\
a_{x L \text { inear }} \\
a_{d \text { Constant }} \\
a_{h \text { Constant }}
\end{array}\right)
\end{aligned}
$$

In summary, the full-field aberration maps for astigmatism and coma can thus be characterized by two numbers, characterizing the optical design $\left(a_{\text {quadratic }}\right.$ and $\left.c_{\text {linear }}\right)$, and by six numbers characterizing misalignment effects $\left(a_{h \text { Constant }}\right.$, $a_{d \text { Constant }}, a_{x L i n e a r}, a_{y L i n e a r}, c_{x \text { Constant }}$, and $\left.c_{y \text { Constant }}\right)$.

\section{RESULTS}

\subsection{OTF-based vs. Shack-Hartmann aberration measurement}

In this section, we present results on the through-focus OTF-based aberration measurement, which was recently proposed by us [1] for two objective lens-tube lens assemblies. We use an 8-year old Olympus 20×/0.75 objective lens, which we know has become significantly aberrated in the course of time, and two simple tube lens designs: a single achromat (Thorlabs AC508-250-A-ML, $250 \mathrm{~mm}$ focal length) and double back-to-back achromat design (two Thorlabs AC508-500-A, $500 \mathrm{~mm}$ focal length). The method is based on measurement of the through-focus Modulation Transfer Function (MTF) and Phase Transfer Function (PTF) obtained from the edge response of a custom-made resolution target, composed of sagittal and tangential edges. This enables quantitative analysis of a number of primary aberrations. The curvature of the best focus as a function of spatial frequency is indicative for spherical aberration, the argument of the OTF quantifies coma, and the best focus as a function of field position for sagittal and tangential edges allows assessment of astigmatism and field curvature. Aberrations are reported here as standard Zernike values, i.e. equal to the Root Mean Square (RMS) value in magnitude, but with the sign of the Zernike fringe coefficient.

Figure 3 and 4 show the results of the measurements with a wavelength around $565 \mathrm{~nm}$. The measured quadratic astigmatism coefficient with the OTF based method are $a_{\text {quadratic }}=-1.44 \lambda / \mathrm{mm}^{2}$ for the single achromat case and $a_{\text {quadratic }}$ $=-0.55 \lambda / \mathrm{mm}^{2}$ for the double back-to-back achromat case, which is a 2.6 times decrease. The constant astigmatism coefficient was $a_{h \text { Constant }}=78.5 \mathrm{~m} \lambda$ for the single achromat case and $a_{h \text { Constant }}=68.7 \mathrm{~m} \lambda$ for the double achromat case. The linear astigmatism coefficient was estimated as $a_{y L i n e a r}=-30.6 \mathrm{~m} \lambda / \mathrm{mm}$ for the single achromat case and $a_{y L i n e a r}=$ $16.4 \mathrm{~m} \lambda / \mathrm{mm}$ for the double achromat case. Clearly, the misalignment related astigmatism coefficients do not change appreciably with tube lens design. The linear and constants coma coefficients of the nodal aberration theory are determined from the coma values measured by the OTF-based method at five points along the field direction (see Figure 
5 and 6). We use a weighted least-squares fit with the inverse of the measurement error squared as weight. For the single achromat case we estimated $c_{\text {linear }}=194 \pm 23 \mathrm{~m} \lambda / \mathrm{mm}, c_{x \text { Constant }}=-137 \pm 9 \mathrm{~m} \lambda$, and $c_{y \text { Constant }}=24 \pm 6 \mathrm{~m} \lambda$, and for the double achromat case $c_{\text {linear }}=222 \pm 32 \mathrm{~m} \lambda / \mathrm{mm}, c_{x \text { Constant }}=-138 \pm 13 \mathrm{~m} \lambda$, and $c_{y \text { Constant }}=22 \pm 9 \mathrm{~m} \lambda$. The comatic aberration coefficients do not change significantly, as opposed to the astigmatic aberration coefficients. It should be mentioned that the measurement of relatively high coma values (higher than about $120 \mathrm{~m} \lambda$ ) from the PTF can be inaccurate and that the estimation of the nodal aberration coefficients from the PTF-based coma values is rather sensitive to the details of the fitting procedure. For example, estimating $c_{\text {linear }}$ for the single achromat case with three field points gives $239 \pm 32 \mathrm{~m} \lambda$, and for five field points with the MTF as weight instead of the inverse squared error gives $181 \pm 7 \mathrm{~m} \lambda$.
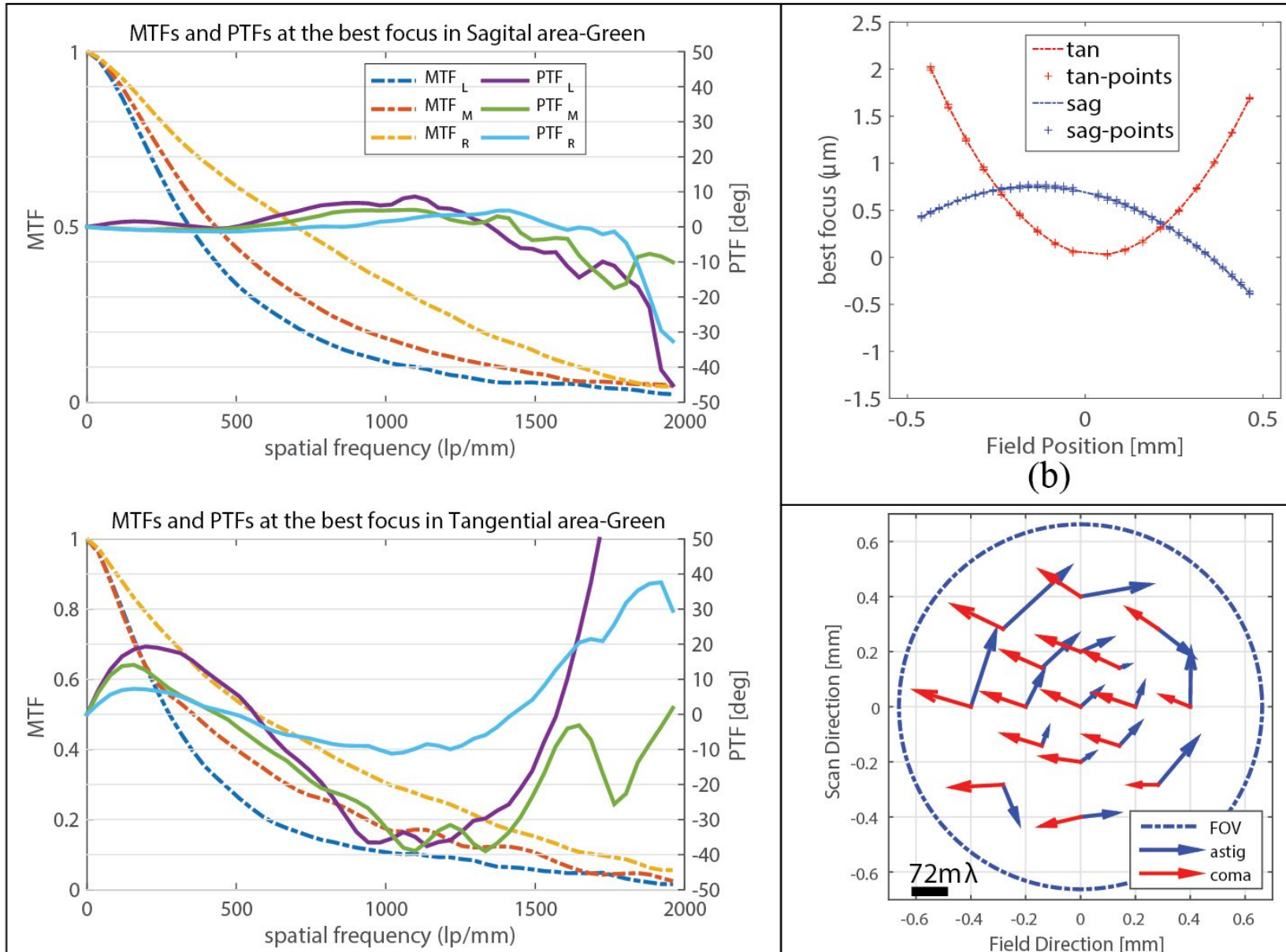

(a)

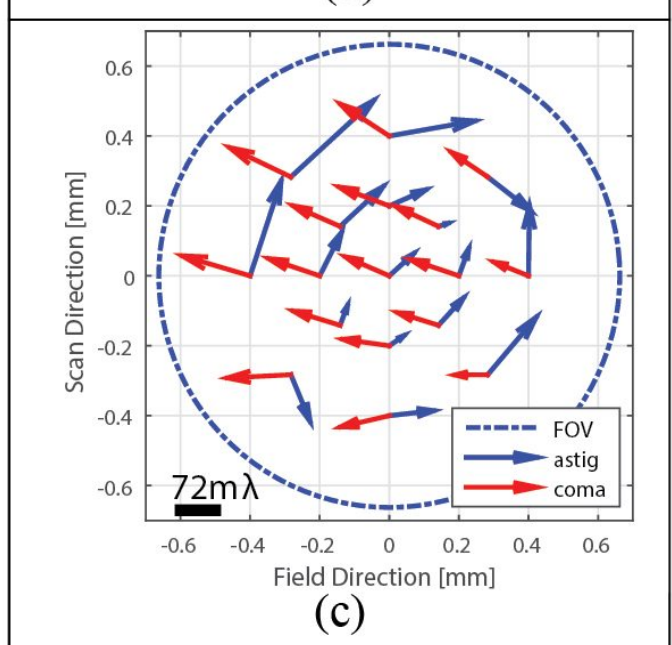

Figure 3. Results for the Olympus $20 \times / 0.75$ objective lens with single achromat tube lens. (a) MTFs (dashed) and PTFs (solid) at the overall best focus along field direction. Subscripts L, M, R denote the left, middle and right side of the FOV and correspond to positions $-0.4,0$ and $0.4 \mathrm{~mm}$ in the field direction. (b) Measured field curvatures. (c) Full-field aberration map measured by Shack-Hartmann sensor. 


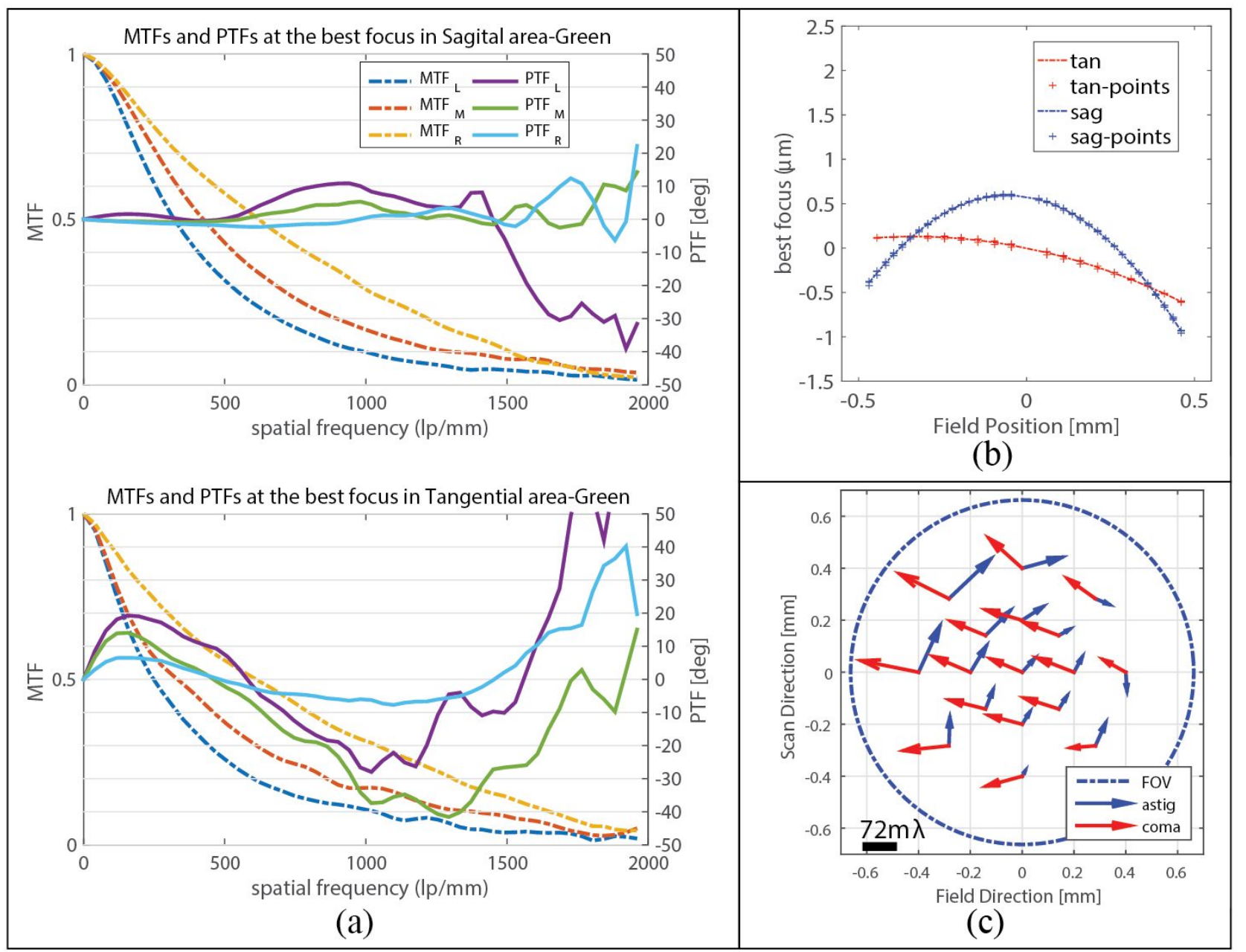

Figure 4. Results for the Olympus 20×/0.75 objective lens with double back-to-back achromat tube lens. (a) MTFs (dashed) and PTFs (solid) at the overall best focus along field direction. Subscripts L, M, and R denote the left, middle and right side of the FOV and correspond to positions $-0.4,0$ and $0.4 \mathrm{~mm}$ in the field direction. (b) Measured field curvatures. (c) Fullfield aberration map measured by the Shack-Hartmann sensor.
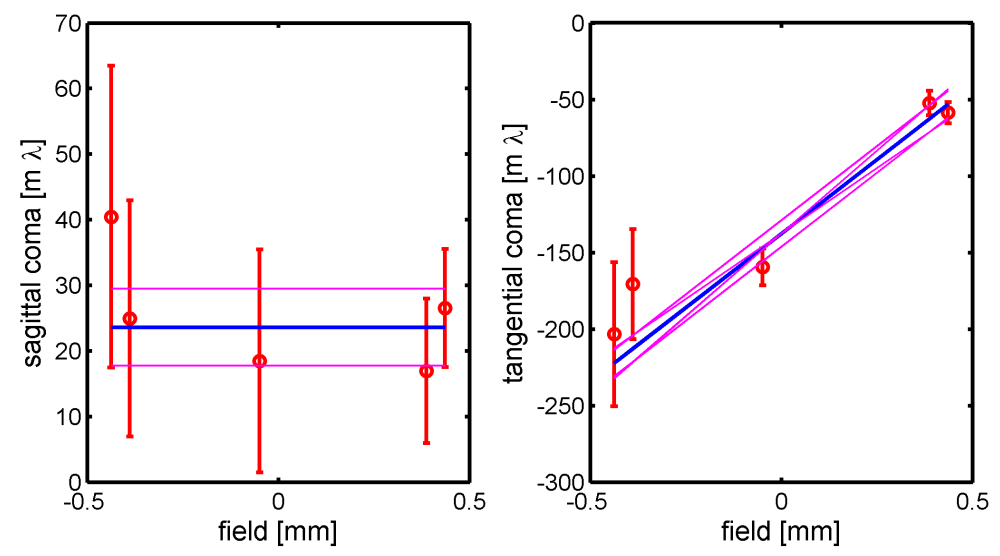

Figure 5. Measured sagittal and tangential coma (red) for the single achromat tube lens design using the nodal aberration theory (blue). The magenta lines indicate the error to the fitted curves. 

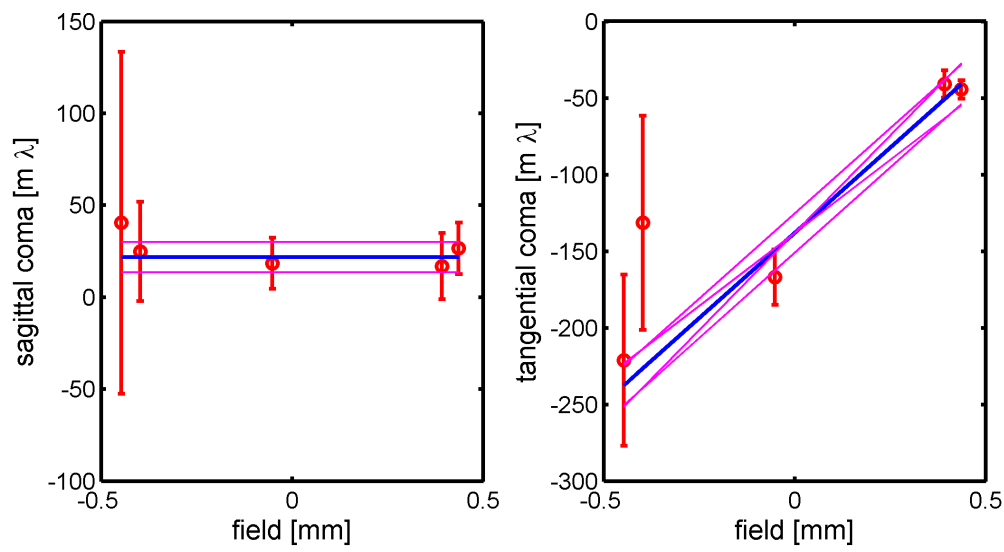

Figure 6. Measured sagittal and tangential coma (red) for the double back-to-back achromat tube lens design using the nodal aberration theory (blue). The magenta lines indicate the error to the fitted curves.

\subsection{Full-field aberration map analysis}

We have analyzed the full-field aberration maps for the two tube lens designs (see Figure 7 and 8 ). We found that the description of the maps using the approach of Thompson, Tessieres and others is quite satisfactory, as the residuals of the fitted map are quite small. It is also apparent from the decomposition of the maps in constant, linear and quadratic contributions that a significant component of the overall level of astigmatism and coma is due to misalignment. These misalignment contributions (constant coma, constant astigmatism, linear astigmatism) do not differ substantially between the two tube lens cases, indicating that the root cause of these aberrations is a misalignment within the lens assembly of the objective lens. The effect of tube lens design shows up in the quadratic astigmatism component, which is substantially reduced in the double achromat design $\left(a_{\text {quadratic }}=-0.37 \mathrm{\lambda} / \mathrm{mm}^{2}\right)$ compared to the single achromat design $\left(a_{\text {quadratic }}=-0.89 \mathrm{\lambda} / \mathrm{mm}^{2}\right)$, which amounts to a 2.4 times reduction. The relative improvement of the quadratic astigmatism coefficient is in good agreement with the OTF based method, but the absolute values differ by $50-60 \%$. The coma coefficients are for the single achromat case: $c_{\text {linear }}=89 \mathrm{~m} \lambda / \mathrm{mm}, c_{x \text { Constant }}=-98 \mathrm{~m} \lambda$, and $c_{y \text { Constant }}=30 \mathrm{~m} \lambda$, and for double achromat case: $c_{\text {linear }}=99 \mathrm{~m} \lambda / \mathrm{mm}, c_{x \text { Constant }}=-99 \mathrm{~m} \lambda$, and $c_{y \text { Constant }}=31 \mathrm{~m} \lambda$. Clearly, these values do not change significantly for the two tube lens designs, which is in agreement with the OTF-based measurements. However, the coefficients themselves do differ substantially between the two methods. The constant coma coefficients agree reasonable well, but the linear coma coefficient, however, is a factor 2.1 larger for the OTF-based method.

We also measured and analyzed the full-field aberration maps for cases with intentional misalignment using the single achromat tube lens design. In particular, we have used a decenter of the objective lens of $\sim 3 \mathrm{~mm}$ along the field direction of the scanner and a tilt of $\sim 2.5 \mathrm{mrad}$ of the objective lens around an axis along the scan direction of the scanner. The amount of tilt we could add is restricted by the mechanical mount of both the objective lens and the Shack-Hartmann sensor. The results are shown in Figure 9 and 10 for coma and astigmatism respectively. The estimated linear coma coefficient does not change considerably due to misalignment: $c_{\text {linear }}=89,79$ and $99 \mathrm{~m} \lambda / \mathrm{mm}$ for the aligned, decentered and tilted cases, respectively, which gives coma of 36,32 and $40 \mathrm{~m} \lambda$ at $0.4 \mathrm{~mm}$ away from the center of the FOV. The estimated quadratic astigmatism coefficient also does not vary considerably: $a_{\text {quadratic }}=-0.89,-0.91$ and $-0.88 \lambda / \mathrm{mm}^{2}$ for the aligned, decentered and tilted cases, respectively, which gives astigmatism equal to 143,146 , and $140 \mathrm{~m} \lambda$ at $0.4 \mathrm{~mm}$ field position. The constant coma coefficients $\left(c_{x \text { Constant }}, c_{y \text { Constant }}\right)$ are $(-98,30),(-141,50)$, and $(-109,27) \mathrm{m} \lambda$ for the aligned, decentered and tilted cases, respectively, which gives 103,149, and $112 \mathrm{~m} \lambda$ with an average angle of about 163 degree. This indicates that decenter has a significant impact on the constant coma coefficients $c_{x \text { Constant }}$ and $c_{y \text { Constant }}$. The constant astigmatism coefficients $\left(a_{h \text { Constant }}, a_{d \text { Constant }}\right)$ are $(-8,54),(-6,66)$, and $(-25,68) \mathrm{m} \lambda$ for the aligned, decentered and tilted cases, respectively, which gives 55,66 , and $73 \mathrm{~m} \lambda$ with an average angle of 51 degree. This implies that the used misalignments do not impact these coefficients significantly. The linear astigmatism coefficients $\left(a_{x L i n e a r}, a_{y L \text { inear }}\right)$ are $(43,-117),(4,-93)$, and $(232,-118) \mathrm{m} \lambda / \mathrm{mm}$ for the aligned, decentered and tilted cases, respectively. Apparently, decenter has a minor impact on astigmatism and tilt has a major impact on astigmatism. Moreover, the effect is largely restricted to the coefficient $a_{x L i n e a r}$, which agrees with the direction of tilt. The averaged residual coma over the FOV is 9 , 30 , and $11 \mathrm{~m} \lambda$ and the averaged residual astigmatism over the FOV is $8,12,13 \mathrm{~m} \lambda$ for the aligned, decentered and tilted cases, respectively. This implies that the experimental data is well described by the nodal aberration theory. 


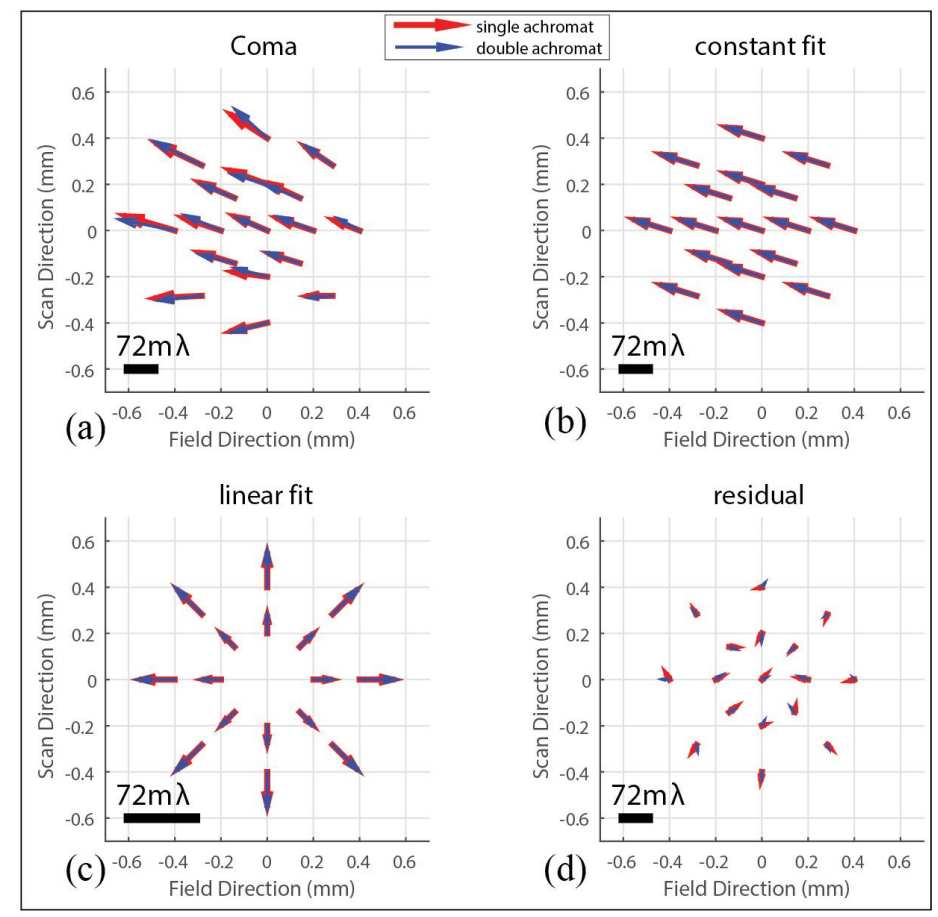

Figure 7. Full-field coma map for the Olympus $20 \times / 0.75$ objective lens with either the single achromat (red arrows) or the double back-to-back achromat (blue arrows) tube lens. The measured coma map (a) is decomposed to its components: (b) constant coma fit, (c) linear coma fit, and (d) residual coma.

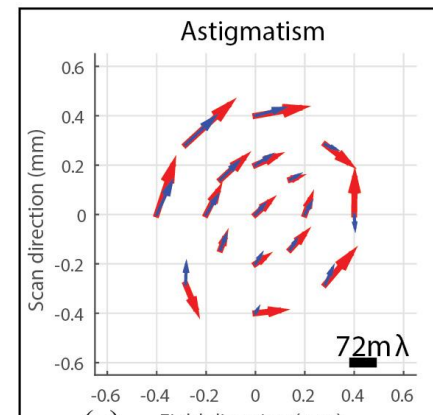

(a) Field direction $(\mathrm{mm})$

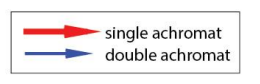

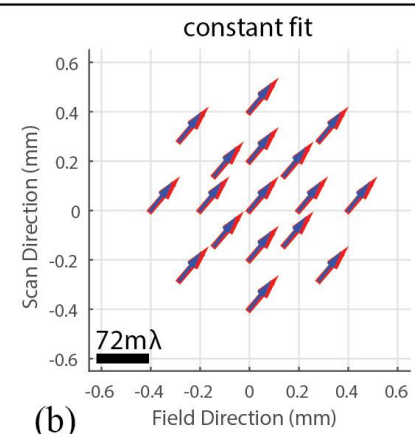

(b)

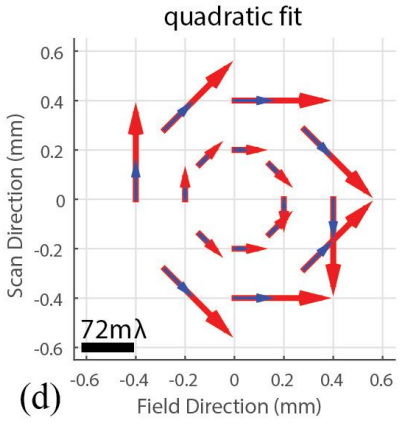

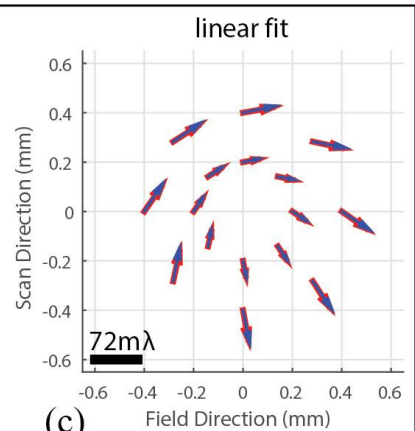

(c)

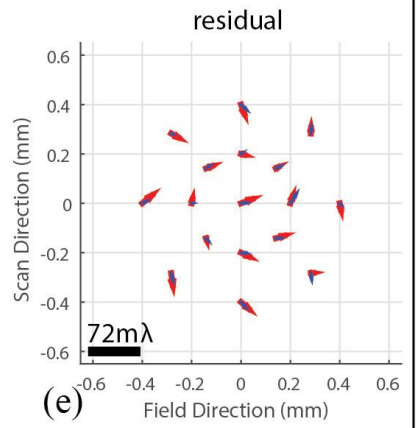

Figure 8. Full-field astigmatism map for the Olympus $20 \times / 0.75$ objective lens with either the single achromat (red arrows) or the double back-to-back achromat (blue arrows) tube lens. The measured astigmatism map (a) is decomposed to its components: (b) constant astigmatism fit, (c) linear astigmatism fit, (d) quadratic astigmatism fit, and (e) residual astigmatism. 


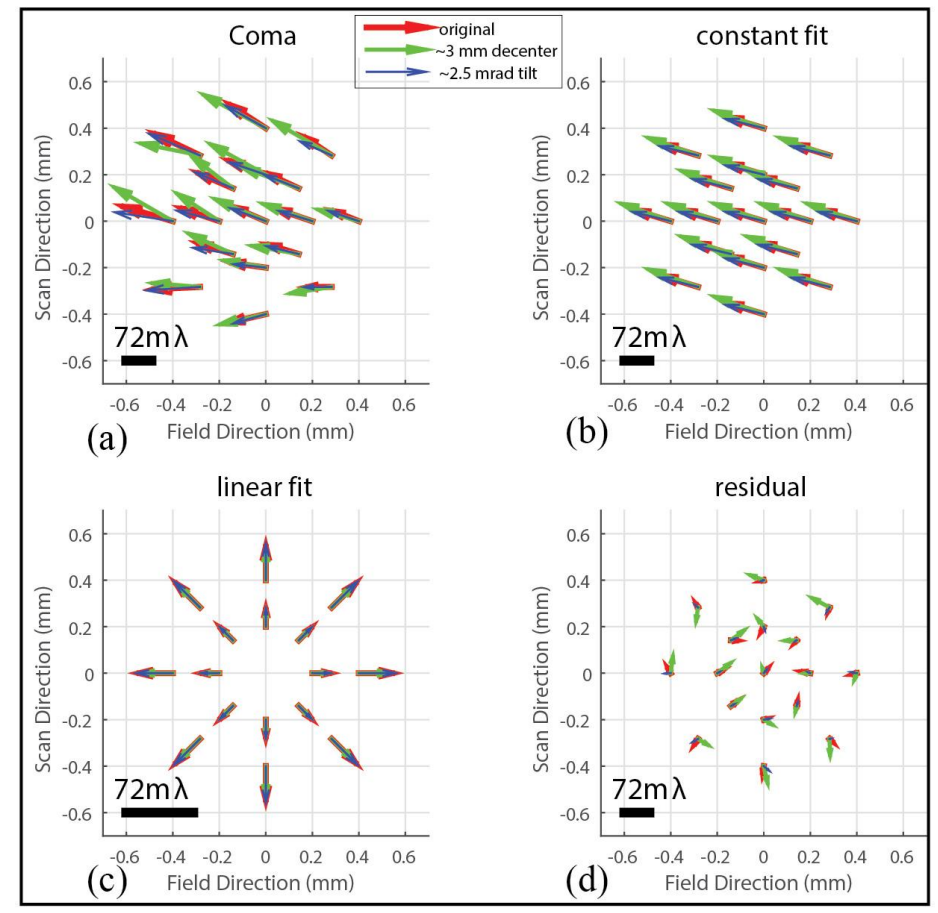

Figure 9. Full-field coma map for the Olympus $20 \times / 0.75$ objective lens with the single achromat (red arrows: original alignment, green arrows: $\sim 3 \mathrm{~mm}$ decenter of objective lens along field position to the left, blue arrows: $\sim 2.5 \mathrm{mrad}$ tilt of objective lens around an axis along the scan direction of the scanner). The measured coma map (a) is decomposed to its components: (b) constant coma fit, (c) linear coma fit, and (d) residual coma.

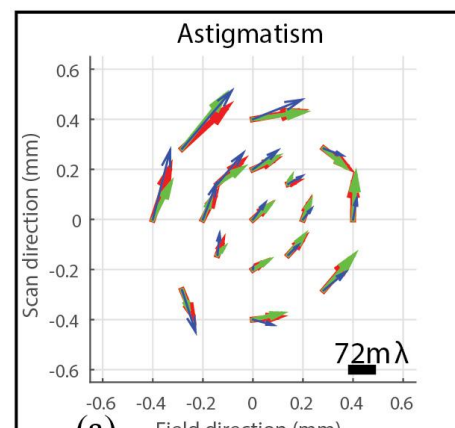

(a) Field direction $(\mathrm{mm})$

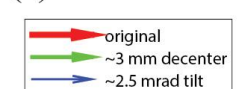

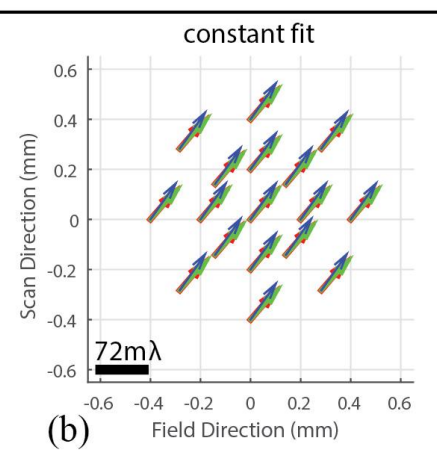

(b)

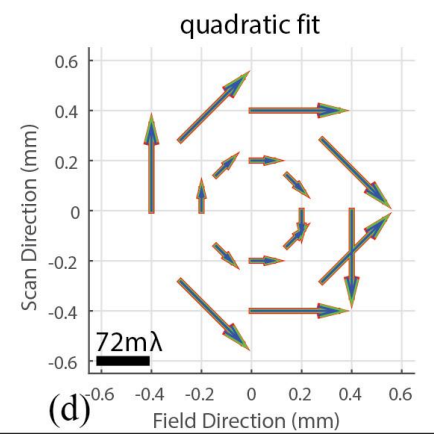

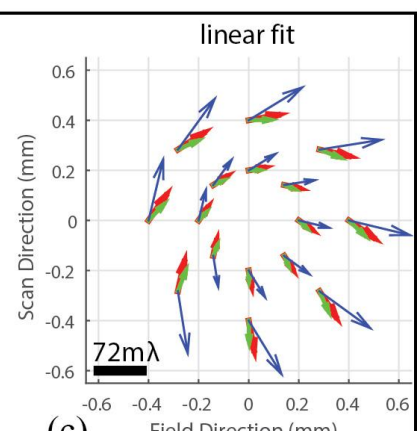

(c) Field Direction (mm)

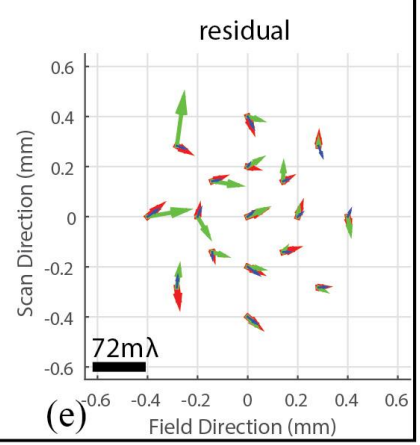

Figure 10. Full-field astigmatism map for the Olympus 20×/0.75 objective lens with the double back-to-back achromat (red arrows: original alignment, green arrows: $\sim 3 \mathrm{~mm}$ decenter of objective lens along field position to the left, blue arrows: $\sim 2.5$ mrad tilt of objective lens around an axis along the scan direction of the scanner). The measured astigmatism map (a) is decomposed to its components: (b) constant astigmatism fit, (c) linear astigmatism fit, (d) quadratic astigmatism fit, and (e) residual astigmatism. 


\section{CONCLUSION}

We have measured full-field aberration maps of WSI system by using a Shack-Hartmann wavefront sensor setup. These maps have been analyzed with nodal aberration theory in order to disentangle aberrations that are inherent to the optical design from aberrations that arise from misalignment. We have restricted the analysis to the lowest order astigmatism and coma. For these two types of aberrations two coefficients quantify the level of aberrations from design and another six coefficients are a measure of the composite level of misalignment. We have experimentally compared two different tube lens designs and confirmed that only the optical design related astigmatism coefficient differs. Intentional decenter of the objective lens predominantly impacts the misalignment related coefficients for constant coma, and intentional tilt of the objective lens has a significant effect on the misalignment related coefficients for linear astigmatism. The full-field aberration map analysis we describe may be used to track root causes of aberrations that are revealed by e.g. throughfocus OTF-based measurement, and in this way assess the optical quality of individual optical components such as

objective lenses or tube lenses, or to improve the alignment of the different optical components within a WSI system.

\section{REFERENCES}

[1] S. M. Shakeri, B. Hulsken, S. Stallinga et al., "Optical Quality Assessment of Whole Slide Imaging Systems for Digital Pathology," Optics Express, 23(2), 1319-1336 (2015).

[2] B. C. Platt, and R. Shack, "History and principles of Shack-Hartmann wavefront sensing," J Refract Surg, 17(5), S573-S577 (2001).

[3] R. Tessieres, "Analysis for alignment of optical systems", MSc dissertation (University of Arizona, Tucson, 2003).

[4] P. L. Schechter, and R. S. Levinson, "Generic misalignment aberration patterns in wide-field telescopes," Publications of the Astronomical Society of the Pacific, 123(905), 812-832 (2011).

[5] R. V. Shack, and K. Thompson, "Influence of alignment errors of a telescope system on its aberration field," Proc. SPIE 0251, 146-153 (1980).

[6] J. Wang, B. H. Guo, Q. Sun et al., "Third-order aberration fields of pupil decentered optical systems," Optics Express, 20(11), 11652-11658 (2012).

[7] K. Thompson, "Description of the third-order optical aberrations of near-circular pupil optical systems without symmetry,” J. Opt. Soc. Am. A, 22(7), 1389-1401 (2005).

[8] K. P. Thompson, T. Schmid, O. Cakmakci et al., "Real-ray-based method for locating individual surface aberration field centers in imaging optical systems without rotational symmetry," J. Opt. Soc. Am. A, 26(6), 1503-1517 (2009).

[9] K. P. Thompson, T. Schmid, and J. P. Rolland, "Recent Discoveries from Nodal Aberration Theory," International Optical Design Conference, 7652, (2010).

[10] A. M. Manuel, "Field-Dependent Aberrations for Misaligned Reflective Optical Systems", PhD dissertation (Universiy of Arizona, Arizona, 2009). 